\title{
Host and pathogen specific biomarkers for melioidosis disease management
}

\author{
M Natesan
}

Fluctuations in the composition or abundance of analytes in biological fluids can provide valuable information for diagnosis of infection and monitoring disease progression. Towards this purpose, our laboratory has developed a proteomics workflow for identifying lead biomarkers of melioidosis. First, we utilized a multiplexed microarray constructed with more than 300 recombinant proteins derived from Burkholderiamallei, B. pseudomallei and B. thailandensis. Recombinant Burkholderia proteins (>300) were produced by E. coli expression system. The proteins were printed on nitrocellulose-coated slides using an ink-jet printer. The arrays were probed with serum samples from non-human primates (NHPs) exposed to B. mallei and human melioidosis cases. Elevated antibody responses to specific bacterial proteins were observed in post-exposure serum samples compared to pre-exposure or controls.

Further analysis of the microarray results revealed several candidate pathogen proteins that are potential biomarkers of immune responses. We also used imaging mass spectrometry (iMS) in tandem with laser-capture microdissection and LC-MSMS to identify candidate biomarkers that were present in tissues from primate disease models. The primary MS data were obtained from standard formalin-fixed and paraffin-embedded tissue sections that were processed for optical microscopy. Host and pathogen proteins that were significantly perturbed by infection were identified by comparisons with control tissues.

Calprotectin (CALP), a calcium and zinc binding protein, was elevated in serum of NHPs infected with $B$. mallei, and additional host biomarkers were also enriched within infected tissues. We further examined correlations between CALP and antibiotic treatment outcomes of melioidosis patients. Serum levels of CALP and C-reactive protein (CRP) were significantly higher in melioidosis and non-melioidosis sepsis patients compared to healthy controls. Median CALP levels were higher in melioidosis compared to non-melioidosis sepsis patients, whereas CRP levels were similar in both cases. Notably, intensive intravenous antibiotic treatment of melioidosis patients resulted in lower levels of CALP and CRP ( $\mathrm{p}<0.0001)$, coinciding with recovery. The median percent reduction of CALP and CRP was $71 \%$ for both biomarkers following antibacterial therapy. For acute melioidosis compared to sepsis caused by other infections, the area under the curve (AUC) for CALP was 0.75 , and 0.57 for CRP, indicating that CALP is a better prognostic marker for melioidosis compared to CRP. Thus, reductions in serum CALP levels were linked to therapeutic responses to antibiotics. Our results demonstrate that a combination of multidimensional proteomics approach can be used to identify lead biomarkers of infection detectable in serum

Division of Molecular and Translational Sciences, United States Army Research Institute of Infectious Diseases, Frederick, Maryland, USA.

Address for correspondence: Dr Mohan Natesan, Division of Molecular and Translational Sciences, United States Army Research Institute of Infectious Diseases, Frederick, Maryland, USA +17035177083

Email: mohan.natesan.ctr@mail.mil $@$ https://orcid.org/0000-0002-5958-0711 